\title{
Induced CMB quadrupole from pointing offsets
}

\author{
Adam Moss, * Douglas Scott $]^{\dagger}$ and Kris Sigurdson \\ Department of Physics \& Astronomy \\ University of British Columbia, Vancouver, BC, V6T $1 Z 1$ Canada
}

(Dated: August 28, 2018)

\begin{abstract}
Recent claims in the literature have suggested that the WMAP quadrupole is not primordial in origin, and arises from an aliasing of the much larger dipole field because of incorrect satellite pointing. We attempt to reproduce this result and delineate the key physics leading to the effect. We find that, even if real, the induced quadrupole would be smaller than claimed. We discuss reasons why the WMAP data are unlikely to suffer from this particular systematic effect, including the implications for observations of point sources. Given this evidence against the reality of the effect, the similarity between the pointing-offset-induced signal and the actual quadrupole then appears to be quite puzzling. However, we find that the effect arises from a convolution between the gradient of the dipole field and anisotropic coverage of the scan direction at each pixel. There is something of a directional conspiracy here - the dipole signal lies close to the Ecliptic Plane, and its direction, together with the WMAP scan strategy, results in a strong coupling to the $Y_{2,-1}$ component in Ecliptic co-ordinates. The dominant strength of this component in the measured quadrupole suggests that one should exercise increased caution in interpreting its estimated amplitude. The Planck satellite has a different scan strategy which does not so directly couple the dipole and quadrupole in this way and will soon provide an independent measurement.
\end{abstract}

*Electronic address: adammoss@phas.ubc.ca

$\dagger$ Electronic address: dscott@phas.ubc.ca

${ }^{\ddagger}$ Electronic address: krs@phas.ubc.ca 


\section{INTRODUCTION}

Measurements of Cosmic Microwave Background (CMB) anisotropies from space have laid the foundations of the standard model of cosmology. These observations provide prima facie evidence that the Universe is close to spatially flat with nearly scale-invariant initial density fluctuations. It is remarkable that only a handful of other parameters, specifying the fraction of baryonic and dark matter components, together with the local expansion rate and the redshift of reionization, can fit the data over such a wide range of scales.

Despite the success of the standard model (see e.g [1]), cosmologists have searched for evidence of new physics. Unfortunately this practice is fraught with uncertainty because of the prevalence of a posteriori statistics. One particular avenue of investigation has focused on the CMB anisotropies at large angular scales. A low quadrupole, for example, was first noted by the Cosmic Background Explorer (COBE) [2] and subsequently confirmed by the Wilkinson Microwave Anisotropy Probe (WMAP) [3]. Several other features have been discovered in WMAP data (see [4] and references therein), with subsequent debate about their statistical significance. Hence it is crucial to investigate all potential sources of systematic error which could be important at large angular scales.

Recently, it has been claimed that an important effect has been overlooked in the WMAP analysis [5]. This particular issue relates to a $25.6 \mathrm{~ms}$ offset between recording the pointing and differential temperature data from the satellite, which translates into an angular error of about $7^{\prime}$. As part of the WMAP processing pipeline a dipole signal is first removed from the time-ordered data (TOD), and this has a much higher amplitude than the primordial anisotropies. If the pointing is incorrect a residual signal will remain in the TOD, which has been found to induce a quadrupole pattern in the final temperature sky maps. Interestingly, this quadrupole has similar $a_{2 m}$ spherical harmonic coefficients to those of the primordial signal measured by WMAP.

In this short article we show why the claimed result cannot be correct. In doing so we uncover the physics behind the coupling of such systematic effects to certain harmonic modes. We also investigate the implications for the Planck experiment, which measures absolute rather than differential data, and scans the sky very differently to WMAP.

\section{WMAP RESULTS}

The WMAP TOD vector $\mathbf{d}$ can be written as

$$
\mathbf{d}=\mathbf{M t}+\mathbf{n},
$$

where $\mathbf{M}$ is the mapping matrix, $\mathbf{t}$ the sky-map and $\mathbf{n}$ the radiometer noise [6]. Ignoring the noise, the outputs of the two radiometers are [7]

$$
\mathbf{d}=\left(1+x_{\mathrm{im}}\right) \mathbf{i}\left(\hat{\mathbf{n}}_{\mathrm{A}}\right)-\left(1-x_{\mathrm{im}}\right) \mathbf{i}\left(\hat{\mathbf{n}}_{\mathrm{B}}\right),
$$

where $\mathbf{i}$ is the Stokes $I$ parameter and the pointing on the sky is denoted by $\hat{\mathbf{n}}$, with the subscripts indicating the $\mathrm{A}$ and $\mathrm{B}$-side beams. The pre-factor $\left(1 \pm x_{\mathrm{im}}\right)$ for each term is due to transmission imbalance between radiometers, the latest values for which can be found in [8]. These factors are constant in time, and are easily absorbed into the mapping matrix.

For the purposes of this work we assume the noise is zero (including noise would only effect the statistics of the results, not the general features). The maximum likelihood skymap solution is then $\tilde{\mathbf{t}}=\Sigma \tilde{\mathbf{t}}_{0}$, where $\Sigma=\left(\mathbf{M}^{\mathrm{T}} \mathbf{M}\right)^{-1}$ and the 'iteration-0' map $\tilde{\mathbf{t}}_{0}=\mathbf{M}^{\mathrm{T}} \mathbf{d}$. 

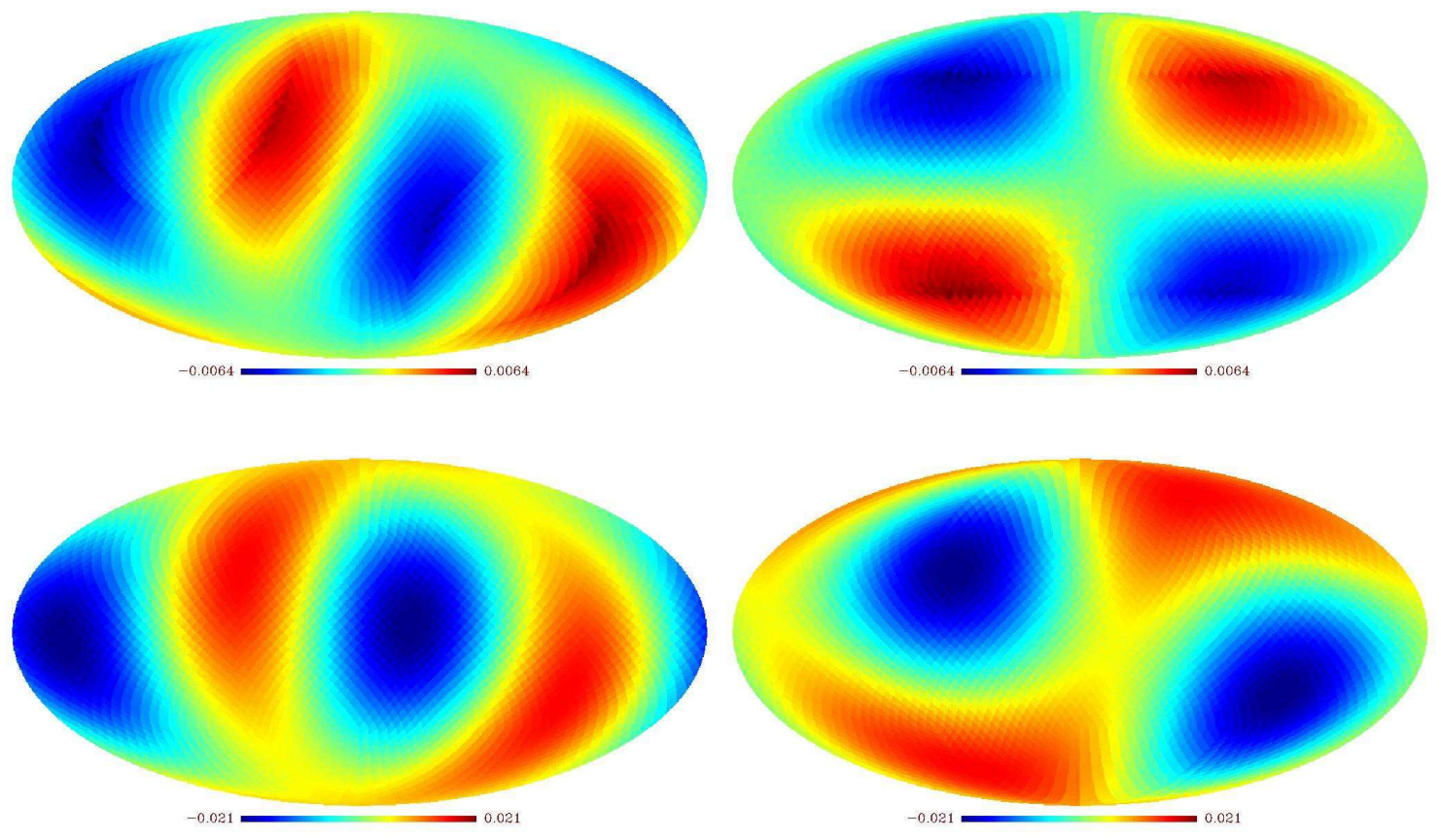

FIG. 1: (Top): Induced quadrupole from a $25.6 \mathrm{~ms}$ pointing offset in Galactic (left) and Ecliptic (right) co-ordinates. (Bottom): WMAP quadrupole derived from the 7-year ILC map in Galactic (left) and Ecliptic (right) co-ordinates. Units are in $\mathrm{mK}$ for each map. The bottom panels are strongly correlated with the upper ones, although with an amplitude about 3 times higher.

The mapping matrix $\mathrm{M}$ has $N_{\mathrm{t}}$ rows and $N_{\mathrm{p}}$ columns, where $N_{\mathrm{t}}$ is the number of time samples for each radiometer and $N_{\mathrm{p}}$ the number of pixels in the map.

To study the effects of a pointing offset, we assume our TOD contains a pure dipole signal. We fix the barycentric component to an amplitude $3.3463 \mathrm{mK}$ with Galactic coordinates $(l, b)=\left(263.87^{\circ}, 48.2^{\circ}\right)[9]$. With a CMB monopole temperature of $T_{0}=2.725 \mathrm{~K}$, this corresponds to a Galactic velocity $\mathbf{v}_{\text {gal }}=(-26.2,244.0,274.4) \mathrm{km} \mathrm{s}^{-1}$. We also include an additional varying dipole component due the satellite motion around the Sun, such that the total velocity is $\mathbf{v}=\mathbf{v}_{\text {gal }}+\mathbf{v}_{\text {sol }}$. The dipole at each pointing is then $\left(T_{0} / c\right) \mathbf{v} \cdot \hat{\mathbf{n}}$ in temperature units.

From this simulated time-stream we then remove the dipole evaluated at a small pointing offset (corresponding to the claimed timing discrepancy), leaving a residual signal in the TOD. We next run our map-making code, solving for the sky-map by a conjugate gradient method. We produce maps at a HEALPIX [10] resolution of nside $=16$.

Maps at W-band, which contain 30 observations (each lasting $51.2 \mathrm{~ms}$ ) per 1.536 second 'science frame', are shown in Fig. 1. In this case the central point of each observation corresponds to the claimed offset between recording the pointing information and recording the differential data from the satellite [5]. For W-band we can therefore use available WMAP software [15] to compute the pointing offset, as the code also gives the interpolated pointing at the centre of each observation. The resulting map is characterized by a quadrupole with an amplitude $\sim 6.5 \mu \mathrm{K}$, and in Ecliptic coordinates is close to a pure $Y_{2,-1}$ spherical harmonic mode. For comparison, we also plot in Fig. 1 (bottom panels) the WMAP quadrupole derived from the 7-year internal linear combination (ILC) map. 

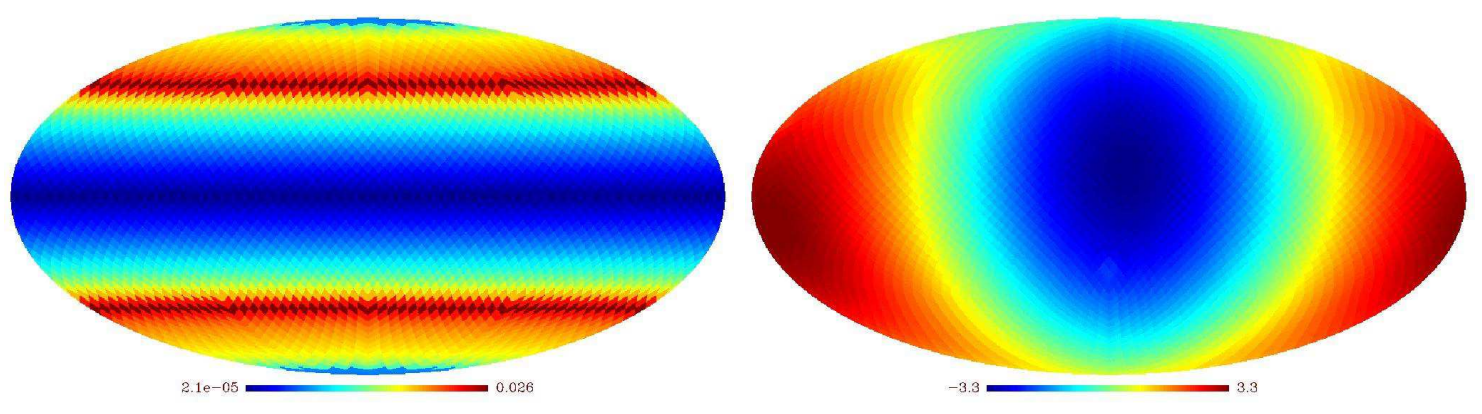

FIG. 2: Modulus of the scan tangent vector expectation value for WMAP (left), along with the underlying dipole field (right, units are $\mathrm{mK}$ ), in Ecliptic co-ordinates.

The induced quadrupole maps are similar in structure to the published WMAP quadrupole. However, we note that we obtain an amplitude lower than that claimed in [5], by approximately a factor of 3 . Hence, even if the claimed effect was real it could not have a large enough amplitude to explain the WMAP result. Nevertheless, the fact that it would produce a quadrupole so similar is intriguing and seems worthy of further investigation.

\section{UNDERSTANDING THE INDUCED QUADRUPOLE}

To make sense of this effect we built a toy model of the scanning strategy for satellites such as WMAP and Planck. The pointing vector can be obtained as a function of time by orienting the satellite focal plane $(\mathrm{FP})$ in the $+z_{\mathrm{FP}}$ direction. The beam (or pair of beams in the case of $W M A P)$ is specified by the opening angle $\theta_{\mathrm{b}}$ with respect to the $+z_{\mathrm{FP}}$ direction. In this frame the satellite spins at an angular velocity $\omega_{\mathrm{s}}=2 \pi / t_{\mathrm{s}}$, where $t_{\mathrm{s}}$ is the spin period.

In order to achieve coverage at the Ecliptic poles the focal plane is rotated at a precession angle $\theta_{\mathrm{p}}$ with respect to the anti-solar direction (which is now defined as the new spacecraft (SC) $+z_{\mathrm{SC}}$ direction). The satellite then spins at a rate $w_{\mathrm{p}}=2 \pi / t_{\mathrm{p}}$ in this frame. Finally, these co-ordinates are rotated into the Ecliptic Plane (with $+z$ corresponding to the north Ecliptic pole) and the solar rotation rate is fixed at $w_{\mathrm{r}}$.

This toy model accurately reproduces the pointing of WMAP and Planck. For WMAP 3], the relevant parameters are $\theta_{\mathrm{b}}=70^{\circ}, \theta_{\mathrm{p}}=22.5^{\circ}, t_{\mathrm{s}}=129 \mathrm{~s}, t_{\mathrm{p}}=3600 \mathrm{~s}$, while for Planck 11] they are $\theta_{\mathrm{b}}=85^{\circ}, \theta_{\mathrm{p}}=7.5^{\circ}, t_{\mathrm{s}}=60 \mathrm{~s}$ and $t_{\mathrm{p}}=6$ months. Note that the precession period is much longer for Planck than WMAP.

We can introduce a time lag $\delta t$ to characterize the pointing offset - the residual signal at each pointing is then $\left(T_{0} / c\right) \delta t \mathbf{v} \cdot \partial \hat{\mathbf{n}} / \partial t$. For a single beam experiment such as Planck the zeroth order approximation for the maximum likelihood solution map is the expectation value (in the long-time limit) of the scan tangent vector at each pixel (which we define as $\langle\partial \hat{\mathbf{n}} / \partial t\rangle)$ dotted with the dipole velocity. The overall amplitude is proportional to the spin rate of the satellite.

For an experiment such as $W M A P$ the $\tilde{\mathbf{t}}_{0}$ map is constructed from a difference signal between the two beams, $\left(T_{0} / c\right) \delta t \mathbf{v} \cdot\left[\partial \hat{\mathbf{n}}_{\mathrm{A}} / \partial t-\partial \hat{\mathbf{n}}_{\mathrm{B}} / \partial t\right]$ (ignoring transmission imbalance factors). The maximum likelihood sky-map solution $\tilde{\mathbf{t}}$ is also complicated by the fact that the $\Sigma$ matrix is not diagonal. We tested the implications of this by assuming that WMAP could also operate as a total intensity experiment. 
In the default 'differencing mode' with the $W M A P$ scan pattern and $\delta t=25.6 \mathrm{~ms}$, we recover the induced quadrupole shown in Fig. 1 to good precision. In 'total intensity mode' the induced signal was around a factor of several lower. In this case there is a subdominant contribution from the $Y_{4,-1}$ harmonic, which has an amplitude of around a factor of 4 less than the $Y_{2,-1}$ mode. The reason for these results is that, for $W M A P, \partial \hat{\mathbf{n}}_{\mathrm{A}} / \partial t \simeq-\partial \hat{\mathbf{n}}_{\mathrm{B}} / \partial t$, so the $\tilde{\mathbf{t}}_{0}$ map is similar in both differencing and total intensity modes. The $\tilde{\mathbf{t}}_{0}$ map contains $Y_{2,-1}$ and $Y_{4,-1}$ components, but the latter is suppressed by the map-making operation in the differencing mode.

The overall structure of the final $\tilde{\mathbf{t}}$ maps can therefore be understood by considering $\langle\partial \hat{\mathbf{n}} / \partial t\rangle$ and the underlying barycentric dipole field. We show these quantities in Ecliptic co-ordinates in Fig. 2, which should be compared with the induced map in Fig. 1. Near the Ecliptic poles pixel scans are approximately isotropic, so the sum of dot products with the dipole velocity cancel over the course of a year's observations. Along the Plane, the component of the dipole velocity parallel to the scan direction is small, since pixels are mainly scanned orthogonal to the plane. Moreover, the sum of north/south scans cancel over a year. For angles at around $\theta_{\mathrm{p}}$ from the poles pixels are mainly scanned in a single direction parallel to the equator. Here the dot product with the dipole velocity is large in certain azimuthal directions, resulting in a large induced signal.

We note that it is an unfortunate coincidence that the direction of the dipole field in the Ecliptic Plane results in a quadrupole with approximately the same phase as the primordial signal of $W M A P$. For the WMAP scan strategy the dipole and quadrupole patterns therefore have a special and unfortunate relationship. Although the pointing offset effect appears not to be real, one can think of other systematics which could potentially couple to the large dipole field in a similar way. These include an asymmetric beam or a time constant in the instrument response. Therefore, it seems reasonable that one should take additional care in removal of the dipole from the TOD, since the induced signal would be similar to the primordial quadrupole.

With our toy model in hand, the scan parameters can be adjusted to test the impact of other scan strategies on the induced quadrupole map. For the approach of the Planck satellite the pixel hits map is no longer azimuthally symmetric (after a year, say) due to the long precession period. The modulus of the scan tangent vector, along with the induced signal for a time delay of $\delta t=25.6 \mathrm{~ms}$, are shown in Fig. 3. The amplitude of the map is similar to that obtained for WMAP, but the structure is very different due to the different scanning geometry. Therefore, one positive aspect of the Planck scan strategy is that one would not expect to see similar systematics coupling to the quadrupole for the Planck scan strategy.

An an aside, our toy model can also be useful in evaluating scan parameters of future CMB satellites. For example, the Experimental Probe of Inflationary Cosmology (EPIC) [12] mission proposal requires that pixel scans are as isotropic as possible to minimize polarization angle errors. We find that a spin and precession period similar to $W M A P$, but with $\theta_{\mathrm{b}}=$ $45^{\circ}$ and $\theta_{\mathrm{p}}=45^{\circ}$, would result in improved angular coverage at each pixel compared to $W M A P[13]$.

\section{OBSERVATIONS OF POINT SOURCES}

So far we have focused on the induced signal from the large CMB dipole component. Observations of point sources could also leave an interesting signature, depending on the 

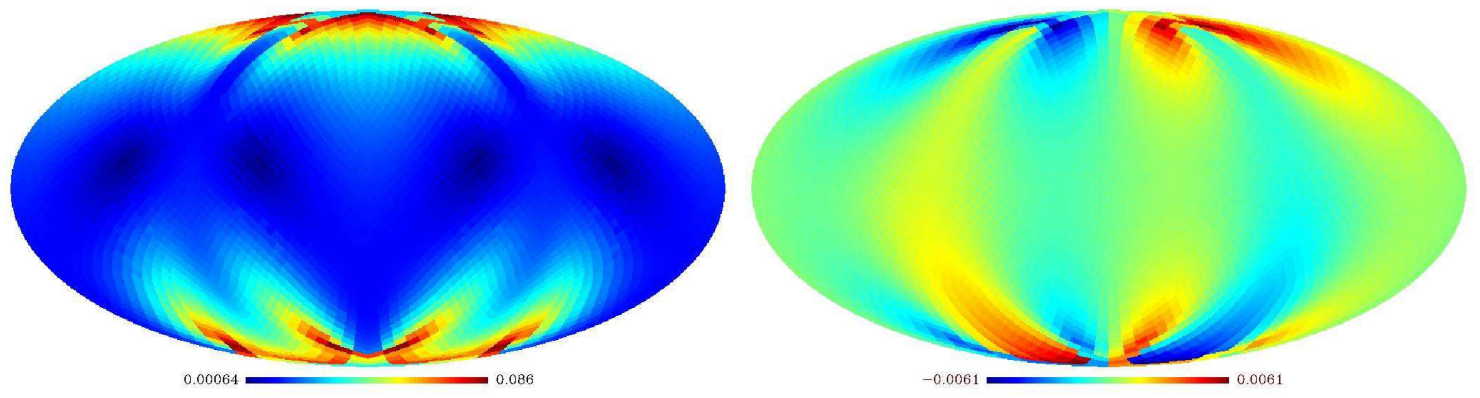

FIG. 3: Modulus of the scan tangent vector expectation value for Planck (left) and the induced map (right, units are $\mathrm{mK}$ ) in Ecliptic co-ordinates.
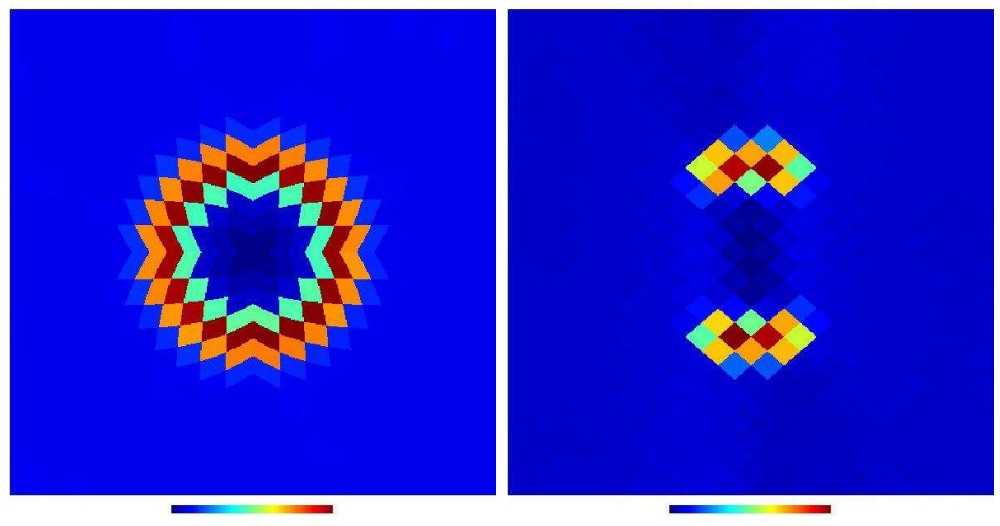

FIG. 4: Observed point source for the $W M A P$ scan pattern, originally at an Ecliptic pole (left) and on the equator (right). We have set the angular pointing error to be larger than the beam size to exaggerate the effect.

instrument beam size compared to the angular pointing error $\theta_{\mathrm{e}}$. In Fig. 4 we show an example of two point sources, one originally located at an Ecliptic pole and one on the equator. We use the WMAP scan strategy and set the pointing error to be larger than the beam size to highlight the effect. At the pole, the source is observed as a circular ring with radius $\theta_{\mathrm{e}}$, while on the equator there are two arcs in the north/south directions. This is consistent with our earlier discussion of the direction of pixel scans at these locations.

In reality, the beam size of $W M A P$ is larger than the claimed pointing offset (for W-band the beam is $\sim 12^{\prime}$ ), so one would observe a smearing of sources rather a distinct offset. We expect this effect would still be detectable since $W M A P$ has a large catalog of $\sim 400$ sources at a signal-to-noise exceeding 5 [14]. Binning sources by Ecliptic latitude and then stacking could lead to tight constraints on timing residuals; however, we leave this study for future work.

Point sources might also leave additional features in the maps if a timing residual was present. When operating in 'total intensity mode', we find no other features in the map apart from those localized around the point source, but in 'difference mode' we find low amplitude stripes in the map at a distance from the source corresponding to twice the beam opening 
angle. These features are only present when there is a timing residual, and presumedly arise from the inability of the maximum likelihood solver to recover a single solution which is actually the difference between two maps. One could imagine in principle adding an additional free parameter to the map-maker to solve for the timing offset. However, since the gross effects of the claimed timing mismatch are not apparent in the data, there seems little point in investigating the more subtle effects.

Finally, we should mention a related issue, which is that a timing offset would be at least partially calibrated out in practice. The WMAP beam boresight is calibrated on twiceyearly observations of Jupiter. Therefore, the timing offset would be degenerate with the offset of a given detector in the focal plane (relative to the boresight) in that particular scan direction. Timing offsets in other scan directions would then only be partially corrected by the Jupiter calibration. This could be easily be accounted for in our code, however due to uncertainty in the precise details of the calibration (e.g. Jupiter is observed for 45 day intervals twice-yearly), we have not explored this correction in more depth.

\section{CONCLUSIONS}

We have investigated recent claims in the literature that the WMAP quadrupole is systematic in origin, arising from an offset between the recording of pointing and temperature data from the satellite. Due to the WMAP scan pattern coupling with the direction of the dipole field, this effect results in a strong $Y_{2,-1}$ mode in Ecliptic co-ordinates, which happens to be similar in phase to the actual quadrupole. We find that the size of the effect for an offset of $25.6 \mathrm{~ms}$ cannot be large enough to match the observed quadrupole. We have also described reasons why the claimed effect is unlikely to be in the WMAP data, in addition to the WMAP team insisting that any error in their timing could not be nearly as large as claimed. Nevertheless, because of the similarity between the induced and primordial quadrupole signals, one should exercise increased caution in interpreting the amplitude of

the primordial component. Planck has a significantly different scan strategy and will soon provide an independent measurement of the quadrupole.

\section{Acknowledgments}

This research was supported by the Natural Sciences and Engineering Research Council of Canada. We thank Kris Gorski, Mark Halpern and Hao Liu for useful discussions.

[1] D. Scott, Can. J. Phys., 84 (2006) 419 astro-ph/0510731.

[2] G. F. Smoot et al., ApJ, 396 (1992) L1.

[3] C. L. Bennett et al., ApJ, 583 (2003) 1 astro-ph/0301158].

[4] C. L. Bennett et al., arXiv:1001.4758.

[5] H. Liu, S. L. Xiong and T. P. Li, (2010) arXiv:1003.1073.

[6] G. Hinshaw et al., ApJS, 148 (2003) 63 astro-ph/0302222.

[7] N. Jarosik et al., ApJS, 170 (2007) 263 astro-ph/0603452.

[8] N. Jarosik et al., (2010) arXiv:1001.4744.

[9] C. L. Bennett et al., ApJS, 148 (2003) 1 [astro-ph/0302207]. 
[10] K. M. Gorski et al., ApJ, 622 (2005) 759 astro-ph/0409513.

[11] J. Tauber et al., submitted to A\&A.

[12] J. Bock et al., CMBPol white paper (2009) arXiv:0906.1188].

[13] K. M. Gorski, private communication (2010).

[14] E. L. Wright et al., ApJS, 180 (2009) 283 arXiv:0803.0577.

[15] Available at http://lambda.gsfc.nasa.gov/product/map/current/m_sw.cfm 